\title{
Cytokeratin Expressing Oncocytic Variant of Gastrointestinal stromal tumor : A Morphological Mimicker of an Epithelial Malignancy
}

\author{
Malini Goswami \\ Department of Oncology, Yenepoya Medical College, Mangalore (India)
}

Keywords: cytokeratin, oncocytic, gastrointestinal stromal tumor

\begin{abstract}
Dear Sir
A 61 year old male presented with abdominal swelling and vague abdominal discomfort since 1 month. His laboratory parameters were within normal limits. CT scan revealed a large, hypervascular, heterogeneously enhancing mass within the abdominal cavity displacing the intestinal loops.
\end{abstract}

Excision of the mass was performed and grossly it was a large well circumscribed mass measuring $16.5 \mathrm{x} 10 \mathrm{x}$ $8 \mathrm{~cm}$, cut section of which was grey white solid with a firm consistency. This mass was attached to the small intestinal loops. Histopathological examination revealed an encapsulated tumor composed of diffuse sheets of polygonal to ovoid cells with well defined cell membranes, abundant pale eosinophilic cytoplasm, centrally placed ovoid nucleus, fine chromatin and small conspicuous nucleoli(figure1). Mitotic activity was 8-10/50hpf. Necrosis was not present. These tumor cells were limited to the submucosa, muscularis propria and serosa of the small intestine. No mucosal involvement was present.

The following differentials were considered: GIST (oncocytic type), metastatic hepatocellular carcinoma, metastatic eosinophilic variant of clear cell RCC, metastatic hurthle cell carcinoma thyroid, metastatic sex cord stromal tumor, melanoma. A wide IHC panel including the markers CD117, CD34, DOG 1, CK, EMA, S100, glypican, PAX 8, inhibin was applied. The tumor cells revealed positivity for, CK (cytoplasmic granular ) CD117, DOG1,CD34 (figure 2:A-D), while negativity for S100, EMA, glypican, PAX 8 and TTF1.A diagnosis of oncocytic variant of gastrointestinal stromal tumor, high risk, was rendered considering the histomorphological as well as immunohistochemistry profile.

What was unique in this case was the morphology: oncocytic tumor cells with abundant eosinophilic cytoplasm mimicking an epithelial malignancy; what was even more mystifying was the cytokeratin expression in tumor cells creating a confusing picture. However DOG1, CD117 and CD34 positivity helped in labelling the neoplasm as oncocytic variant of GIST. The tumor cells may mimic any oncocytic tumor metastasising from different sites like liver, kidney, thyroid gland or testis.
Gastrointestinal stromal tumours (GISTs) are the most common mesenchymal neoplasms of the gastrointestinal tract $^{[1]}$ Most GISTs exhibit a spindled, epithelioid, or mixed (spindle/epithelioid) cell morphology. ${ }^{[1]}$ Oncocytic variant characterised by the presence of abundant mitochondria has been described in literature. ${ }^{[2]} \mathrm{CK}$ expression in GIST is a rare manifestation, that may lead to diagnostic difficulty and errors as they can easily be mistaken for other epithelial or epithelioid mesenchymal tumors. ${ }^{[3]}$ Aberrant expression of $\mathrm{CK}$ is identified to be a consequence of aberrant synthesis of $\mathrm{CK}$ by tumor cells or cross-reactivity to other intermediate filament proteins.

${ }^{\text {[4] }} \mathrm{CK}$ expression in GISTs may be a phenomenon related to tumor progression, akin to meningioma where CKs are preferentially expressed in high grade tumors and malignant melanoma where $\mathrm{CK}$ positivity is perceived more often in metastatic rather than primary melanoma. ${ }^{[5]}$ In such problematic cases, a diagnosis of GIST can be made when DOG1 immunoreactivity or mutation of KIT or PDGFRA are witnessed. ${ }^{[3]}$

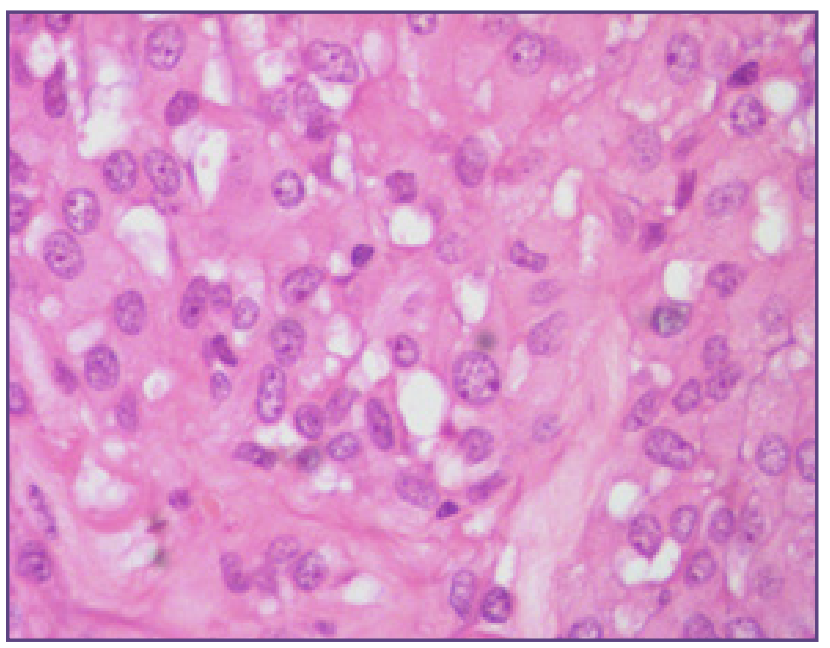

Fig. 1: photomicrograph showing a tumor composed of diffuse sheets ofpolygonal to ovoid cells with well defined cell membranes, abundant pale eosinophilic cytoplasm, centrally placed ovoid nucleus, fine chromatin and small conspicuous nucleoli,H\&E,400X. 


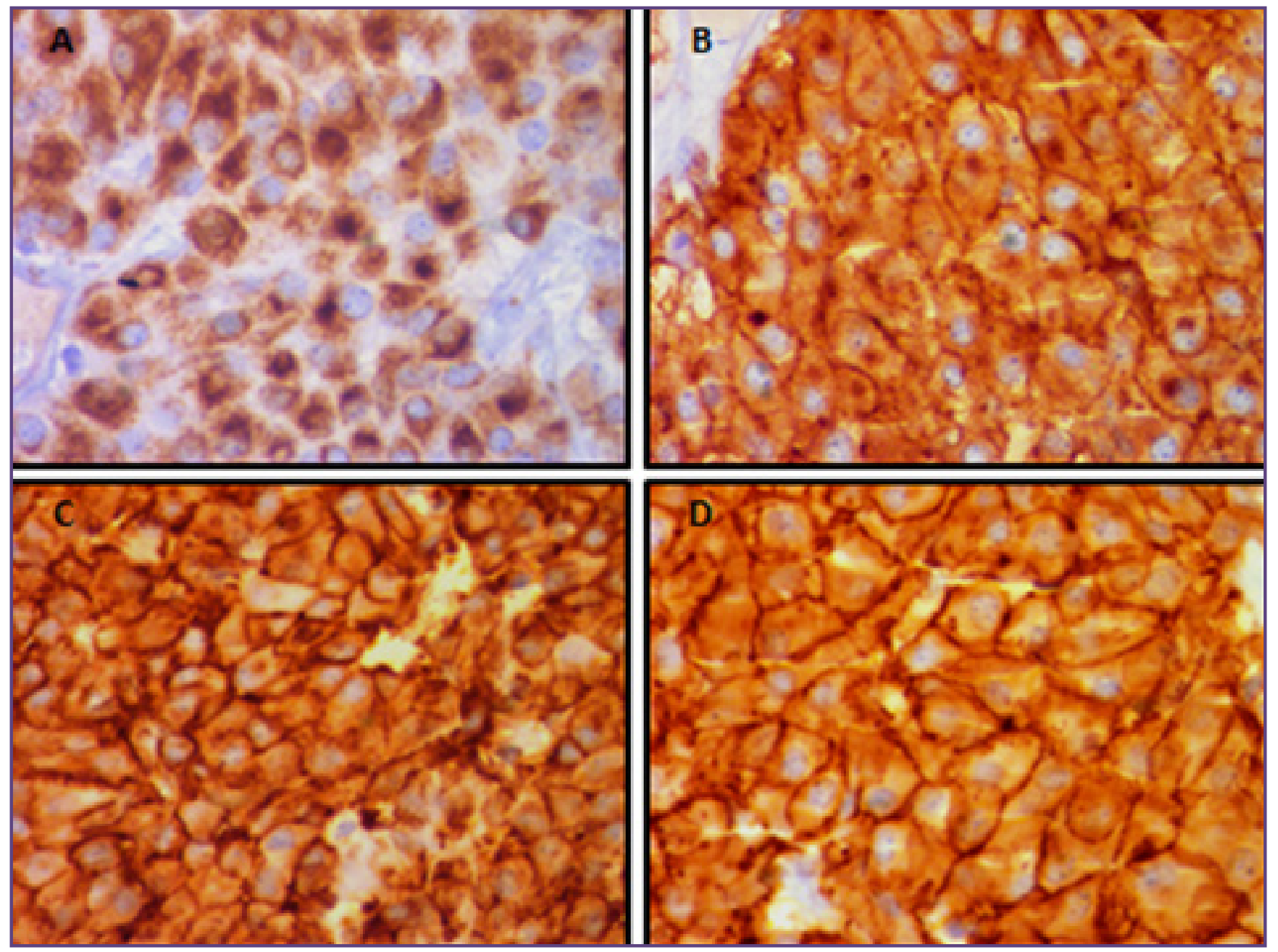

FiG.2:A:photomicrographshowingtumorcellsexpressingcytokeratin(cytoplasmicgranular),CKIHC,400XB:photomicrograph showing tumor cells expressing CD117(cytoplasmic and membranous),CD117 IHC,400X C:photomicrograph showing tumor cells expressing D0G1(cytoplasmic and membranous),D0G1 IHC,400X D:photomicrograph showing tumor cells expressing CD34(cytoplasmic and membranous),CD34 IHC,400X Figure2:A:photomicrograph showing tumor cells expressing cytokeratin(cytoplasmic granular),CK IHC,400X B:photomicrograph showing tumor cells expressing CD117(cytoplasmic and membranous),CD117 IHC,400X C:photomicrograph showing tumor cells expressing DoG1(cytoplasmic and membranous),D0G1 IHC,400X D:photomicrograph showing tumor cells expressing CD34(cytoplasmic and membranous),CD34 IHC,400X.

In conclusion, cytokeratin expression in GISTs more so with an epithelioid/oncocytic morphology is an unwarranted diagnostic pitfall, especially in high grade GISTs with limited biopsy material and from metastatic sites, thereby necessitating the use of molecular analysis or comprehensive immunohistochemistry for difficult cases.

\section{Abbreviations and Symbols}

CT: computed tomography GIST: gastrointestinal stromal tumor RCC: renal cell carcinoma IHC: immunohistochemistry CD: cluster of differentiation DOG1: discovered on GIST CK: cytokeratin S100:100\% soluble in ammonium sulphate at neutral PH PAX 8: paired box gene 8 TTF1: thyroid transcription factor 1 EMA: epithelial membrane antigen PGDFRA: platelet derived growth factor

\section{Acknowledgements}

I would like to thank Dr. Anurag Mehta, who is the head of department, pathology, RGCIRC, for his encouragement and support.

\section{References}

1. Rossi G, Sartori G, Valli R, Bertolini F, Bigiani N,et al. The value of c-kit mutational analysis in a cytokeratin 
positive gastrointestinal stromal tumour. J Clin Pathol 2005;58:991-993.

2. Fülöp E, Marcu S, Milutin D, Borda A. Gastrointestinal stromal tumors: review on morphology, diagnosis and management. Romanian Journal of Morphology and Embryology 2009;50(3):319-326.

3. Kim SS, Choi YD, Lee JH, Choi C. Cytokeratin-Positive Gastrointestinal Stromal Tumor of Biphasic Morphology: A Case Report. Korean J Pathol. 2014; 48(5): 375-378.
4. Fanburg-Smith JC, Majidi M, Miettinen $M$. Keratin expression in schwannoma: a study of 115 retroperitoneal and 22 peripheral schwannomas. Mod Pathol. 2006;19:115-21.

5. Sing Y, Ramdial PK, Ramburan A, Sewram V. Cytokeratin expression in gastrointestinal stromal tumors: morphology, meaning, and mimicry.Indian $\mathrm{J}$ Pathol Microbiol.2014;57(2):209-16.

*Corresponding author:

Dr. Malini Goswami, c/o Pranab Goswami, House number 11, Sundarpur, Bye lane 1(left), RG Barua road, Guwahati-781005, Assam, India Phone: +91 9654488079, 9599875132

Email: malinig87@gmail.com

Date of Submission : 11.12.2016

Financial or other Competing Interests: None. 